\title{
Arriving at the correct taxonomy: a comment on "A new and highly divergent mitochondrial lineage in the Small Five-toed Jerboa, Scarturus elater, from Iran"
}

\author{
Ahmad Mahmoudi ${ }^{1,2^{*}}$ and Boris Kryštufek ${ }^{3}$ \\ ${ }^{1}$ National Reference Laboratory of Plague, Tularemia and Q Fever, Research Centre for Emerging and \\ Reemerging Infectious Diseases, Pasteur Institute of Iran, Akanlu, Kabudar Ahang, Hamadan, Iran \\ ${ }^{2}$ Department of Epidemiology and Biostatistics, Research Centre for Emerging and Reemerging Infectious \\ Diseases, Pasteur Institute of Iran, Tehran, Iran \\ ${ }^{3}$ Slovenian Museum of Natural History, Prešernova 20, SI-1000 Ljubljana, Slovenia \\ *Corresponding author ${ }^{\star}$ : a.mahmoudi.bio@gmail.com
}

Received: 7 January 2020 Accepted: 9 February 2020 Published online: 31 March 2020

\begin{abstract}
We reanalyzed 657 base pairs of mitochondrial cytochrome $b$ (cytb) sequences of small five-toed jerboas Scarturus from Iran, which had been published as Allactaga by Mohammadi et al. (2016) in Zoology in the Middle East. We show that taxonomic names are available for the three main clusters they recognized: S. hotsoni, S. elater, and S. toussi. The last two species each contained two lineages, both in our analysis, as well as in those published earlier. We recommend the taxonomic identification of specimens in molecular analyses and call for deposition of voucher material in responsible public collections.
\end{abstract}

\section{Main Text}

Frequently, the initial step in biological research is identification in which a preexisting taxonomic name is assigned to the individuals of a given species. The name enables one to search for the existing information or to undertake comparisons with other organisms. Accurate species identification is crucial and misidentifications may have major consequences in basic and applied research (Bickford et al., 2007; Costa et al., 2015; Lourenço, 2016; Kryštufek et al., 2019; Taylor et al., 2019). Reference material, either in the form of museum vouchers or deposited nucleotide sequences, is frequently essential for accurate identification.

In a recent work, Mohammadi et al. (2016) readdressed the evolutionary history of small fivetoed jerboas (Allactaga Cuvier, now Scarturus Gloger; see Michaux and Shenbrot, 2017) in Iran by using mitochondrial cytochrome $b$ (cyt $b$ ) marker. They identified five clusters which they either called lineages or clades. Because the nodes in their tree (Figure 2 in Mohammadi et al., 2016) lack information on statistical support, and genuine monophylies are therefore unknown, we shall call them clusters. Mohammadi et al. (2016) concluded that (1) "the clear separation of the mtA lineage from the remaining specimens .... may suggest the occurrence of a new Allactaga species", (2) the remaining clusters match subspecies of A. elater Lichtenstein, and (3) "A. toussi might not be a true species but instead a morphological variant of A. elater". We found their taxonomic interpretation of small five-toed jerboas of Iran surprising and decided to repeat their analyses. We included in our assessment all cyt $b$ sequences used by Mohammadi et al. (2016). To these we added published sequences of Scarturus elater, S. toussi, S. hotsoni Thomas, S. euphratica Thomas, and S. williamsi 
Thomas. A complete list of sequences with GenBank accession numbers and references appears in the Appendix. A number of these sequences, except those from Moshtaghi et al. (2016), are backed by museum vouchers housed in the Zoological Museum of Ferdowsi University of Mashhad, Iran (examined by the first author), Slovenian Museum of Natural History Ljubljana (Boris Kryštufek), General Commission for Scientific Agricultural Research, Damascus, Syria (now most probably lost; examined by Boris Kryštufek), and Department of Biology, Selçuk University, Konya, Turkey (determined by Professor Atilla Arslan). This allowed us to link with great confidence the haplotypes with existing taxonomic names.

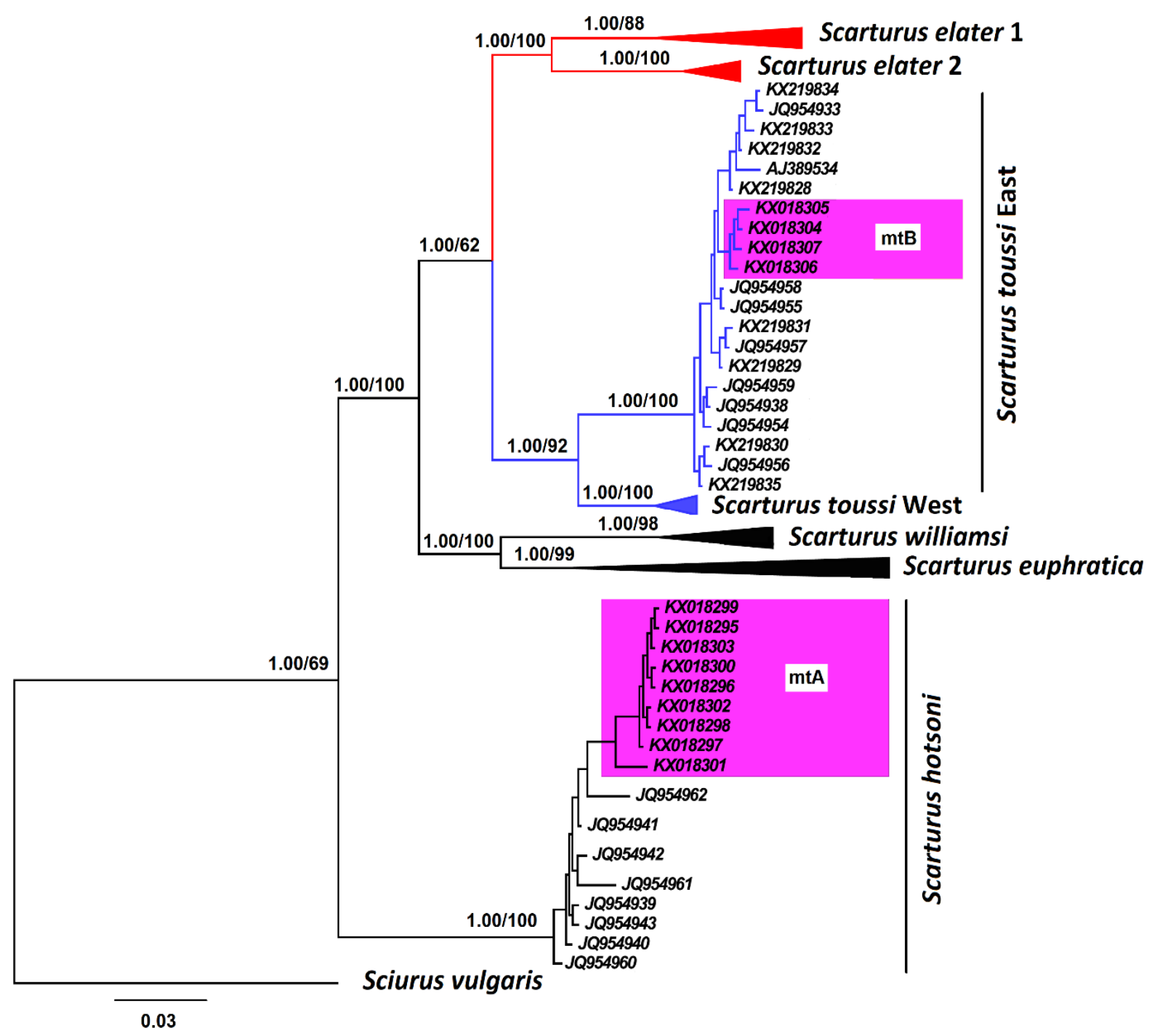

Figure 1: Bayesian Inference tree summarizing phylogenetic relationships among 98 cytochrome $b$ sequences representing all five-toed jerboas (Scarturus) from Iran and adjacent regions. All sequences were downloaded from GenBank (see Appendix for accession numbers). Sequences published by Mohammadi et al. (2016) are labeled as ' $\mathrm{mtA}^{\prime}$ ' and ' $\mathrm{mtB}$ ' and highlight in pink. Taxonomic names for lineages are on the right-hand side and follow Moshtaghi et al. (2016). The first and second numbers on the branches correspond to posterior probability (BPP) and bootstrap (BP) values in the BI and ML tree analyses, respectively. 
Our phylogenetic analysis involved $98 \mathrm{cyt} b$ sequences $(657 \mathrm{bp})$ and followed the protocol described in Moshtaghi et al. (2016). Bayesian Inference (BI) and Maximum Likelihood (ML) analyses gave trees of very similar topologies, therefore only the BI tree is shown in Figure 1. Small five-toed jerboas were in four major lineages which had strong support (BPP=1.0, $\mathrm{BP} \geq 0.88$ ). All sequences from the cluster ' $m \mathrm{AA}^{\prime}$ of Mohammadi et al. (2016) aligned with reference samples of $S$. hotsoni and the cluster ' $\mathrm{mtB}$ ' emerged as identical with ' $S$. toussi East' of Moshtaghi et al. (2016). Furthermore, clusters 6 and 7 of Mohammadi et al. (2016) nested inside'S. elater 2' and 'S. elater 1' of Moshtaghi et al. (2016), respectively. Therefore, the topology of Mohammadi's et al. (2016) tree can be unambiguously interpreted with the existing body of taxonomic knowledge. Moreover, morphological examination of museum vouchers would equally ensure the application of proper taxonomic names, as $S$. hotsoni can be reliably distinguished from other small five-toed jerboas in southwestern Asia by its swollen bullae (Corbet, 1978). Although morphological differences between $S$. elater and $S$. toussi are less prominent, they are stable and allow safe classification of individuals (Darvish et al., 2008). For further phylogeographic structuring of $S$. toussi and $S$. elater, we direct the reader to Dianat et al. (2013), Moshtaghi et al. (2016) and Bannikova et al. (2018).

Palearctic mammals are among the best-known on the planet, and there is a solid foundation for studying their evolution and taxonomy. Despite this, it is imperative that any researcher gain important taxonomic knowledge on the group of interest before any work is undertaken. This should also involve the identification of specimens by traditional morphological characteristics which link modern phylogenetic analysis with the traditional Linnaean taxonomy (Schlick-Steiner et al., 2007).

Finally, we call for long-term preservation of vouchers and their deposition in responsible public collections to enable sound integrative taxonomic studies. Taxonomic results are crucial for species delimitation and all subsequent steps, like mapping distributional ranges, assessing risk for public health and planning conservation management.

\section{Acknowledgements}

This communication is dedicated to late Professor Jamshid Darvish who worked hard to disentangle the taxonomy of Iranian rodents in general and the jerboas in particular. Among other contributions, he also established the Zoological Museum of Ferdowsi University of Mashhad. We would like to thank Atilla Arslan for checking the identity of Scarturus deposited in the Selçuk University, Konya, Christiane Denys (Museum National d'Histoire Naturelle) for thoughtful comments on an earlier draft. Bruce D. Patterson (Field Museum of Natural History) is acknowledged for English editing.

\section{References}

Bannikova, A., Lebedev, V., Dubrovskaya, A., Solovyeva, E., Moskalenko, V., Kryštufek, B., Hutterer, R., Bykova, E., Zhumabekova, B., Rogovin, K. and Shenbrot, G. (2018). Genetic evidence for several cryptic species within the Scarturus elater species complex (Rodentia: Dipodoidea): when cryptic species are really cryptic. Biological Journal of the Linnean Society, 126 (1): 16-39. https://doi.org/10.1093/biolinnean/bly154

Bickford, D., Lohman, D. J., Sodhi, N. S., Ng, P. K. L., Meier, R., Winker, K., Ingram, K. K. and Das, I. (2007). Cryptic species as a window on diversity and conservation. Trends in Ecology and Evolution, 22 (3): 148-155. https://doi.org/10.1016/j.tree.2006.11.004 
Corbet, G. B. (1978). The Mammals of the Palaearctic Region: a taxonomic review. British Museum (Natural History) and Cornell University Press, London and Ithaca (NY). vii + $314 \mathrm{pp}$.

Costa, H., Foody, G. M., Jiménez, S. and Silva, L. (2015). Impacts of species misidentification on species distribution modeling with presence-only data. ISPRS International Journal of Geo-Information, 4 (4): 2496-2518. https://doi.org/10.3390/ijgi4042496

Darvish, J., Hajjar, T., Moghadam Matin, M., Haddad, F. and Akbary Rad, S. (2008). New species of five-toed jerboa (Rodentia: Dipodidae, Allactaginae) from North-east Iran. Journal of Sciences, Islamic Republic of Iran, 19 (2): 103-109.

Dianat, M., Aliabadian, M., Darvish, J. and Akbarirad, S. (2013). Molecular phylogeny of the Iranian Plateau five-toed jerboa, Allactaga (Dipodidae: Rodentia), inferred from mtDNA. Mammalia, 77 (1): 95-103. https://doi.org/10.1515/mammalia-2012-0011

Kryštufek, B., Amori, G. and Chişamera, G. (2019). Taxonomic identification matters: Comment on "Exploring the impact of snow vole (Chionomys nivalis) burrowing activity in the Făgăraș Mountains, Southern Carpathians (Romania): Geomorphic characteristics and sediment budget". Catena, 183. https://doi.org/10.1016/j.catena.2019.104194

Kryštufek, B., Arslan, A., Shehab, A., Abi-Said, M. R., Zupan, S. and Lužnik, M. (2013). Mitochondrial sequences point on a cryptic species in five-toed jerboas, subgenus Paralactaga. Mammalia, 77 (4): 433-438. https://doi.org/10.1515/mammalia-2012-0109

Lourenço, W. R. (2016). Scorpion incidents, misidentification cases and possible implications for the final interpretation of results. Journal of Venomous Animals and Toxins including Tropical Diseases, 22 (1): 21. https://doi.org/10.1186/s40409-016-0075-6

Michaux, J. and Shenbrot, G. (2017). Family Dipodidae (Jerboas), In: Wilson, D. E., Mittermeier, R. A. and Lacher, T. E. (Eds.), Handbook of the Mammals of the World. Volume 7: Rodents II. Lynx Edicions in association with Conservation International and IUCN, Barselona, Spain. pp. 1-30.

Mohammadi, S., Afonso, S., Adibi, M. A., Melo-Ferreira, J. and Campos, R. (2016). A new and highly divergent mitochondrial lineage in the Small Five-toed Jerboa, Allactaga elater, from Iran (Mammalia: Rodentia). Zoology in the Middle East, 62 (3): 206-11. https://doi.org/10.1080/09397140.2016.1202925

Moshtaghi, S., Darvish, J., Mirshamsi, O. and Mahmoudi, A. (2016). Cryptic species diversity in the genus Allactaga (Rodentia: Dipodidae) at the edge of its distribution range. Folia Zoologica, 65 (2): 142-147. https://doi.org/10.25225/fozo.v65.i2.a9.2016

Schlick-Steiner, B. C., Seifert, B., Stauffer, C., Christian, E., Crozier, R. H. and Steiner, F. M. (2007). Without morphology, cryptic species stay in taxonomic crypsis following discovery. Trends in Ecology and Evolution, 8 (22): 391-392. https://doi.org/10.1016/j.tree.2007.05.004

Taylor, P. J., Denys, C. and Fenton, P. D. (2019). Taxonomic anarchy or an inconvenient truth for conservation? Accelerated species discovery reveals evolutionary patterns and heightened extinction threat in Afro-Malagasy small mammals. Mammalia, 83 (4): 313 329. https://doi.org/10.1515/mammalia-2018-0031 
Appendix: Voucher Number/Sample ID, Accession Number, Species, Locality and Reference of the analyzed samples. Note that in quoted sources, including GenBank, all the sequences are labeled by the generic name Allactaga. Collection Acronyms: DBSU Department of Biology, Selçuk University, Konya, Turkey; GCSAR - General Commission for Scientific Agricultural Research, Damascus, Syria; PMS - Slovenian Museum of Natural History, Ljubljana, Slovenia; ZMFUM - Zoological Museum of Ferdowsi University of Mashhad, Mashhad, Iran.

\begin{tabular}{|c|c|c|c|c|}
\hline $\begin{array}{l}\text { Sample ID/ } \\
\text { Voucher } \\
\text { Number }\end{array}$ & $\begin{array}{l}\text { Accession } \\
\text { Numbers }\end{array}$ & $\begin{array}{l}\text { Species/Clades } \\
\text { Moshtaghi et al. } \\
\text { (2016) }\end{array}$ & Locality & Reference \\
\hline- & KX018295 & Scarturus hotsoni & Iran, Damghan, Qusheh & Mohammadi et al. (2016) \\
\hline - & KX018296 & Scarturus hotsoni & Iran, Damghan, Qusheh & Mohammadi et al. (2016) \\
\hline- & KX018297 & Scarturus hotsoni & Iran, Damghan, Qusheh & Mohammadi et al. (2016) \\
\hline- & KX018298 & Scarturus hotsoni & Iran, Damghan, Qusheh & Mohammadi et al. (2016) \\
\hline- & KX018299 & Scarturus hotsoni & Iran, Damghan, Qusheh & Mohammadi et al. (2016) \\
\hline- & KX018300 & Scarturus hotsoni & Iran, Damghan, Qusheh & Mohammadi et al. (2016) \\
\hline- & KX018301 & Scarturus hotsoni & Iran, Damghan, Qusheh & Mohammadi et al. (2016) \\
\hline- & KX018302 & Scarturus hotsoni & Iran, Damghan, Qusheh & Mohammadi et al. (2016) \\
\hline- & KX018303 & Scarturus hotsoni & Iran, Damghan, Qusheh & Mohammadi et al. (2016) \\
\hline ZMFUM2674 & JQ954928 & Scarturus elater 1 & Iran, Golestan & Dianat et al. (2013) \\
\hline ZMFUM2128 & JQ954931 & Scarturus elater 1 & Iran, Kashmar & Dianat et al. (2013) \\
\hline ZMFUM 1429 & JQ954932 & Scarturus elater 1 & Iran, Kashmar & Dianat et al. (2013) \\
\hline ZMFUM1377 & KX219804 & Scarturus elater 1 & Iran, Kashmar & Moshtaghi et al. (2016) \\
\hline ZMFUM1412 & KX219805 & Scarturus elater 1 & Iran, Kashmar & Moshtaghi et al. (2016) \\
\hline ZMFUM2084 & KX219806 & Scarturus elater 1 & Iran, Kashmar & Moshtaghi et al. (2016) \\
\hline ZMFUM1740 & KX219807 & Scarturus elater 1 & Iran, Torbate Heydarieh & Moshtaghi et al. (2016) \\
\hline ZMFUM3542 & KX219808 & Scarturus elater 1 & Iran, Gonabad & Moshtaghi et al. (2016) \\
\hline ZMFUM1905 & KX219809 & Scarturus elater 1 & Iran, Gonbad & Moshtaghi et al. (2016) \\
\hline ZMFUM2704 & KX219810 & Scarturus elater 1 & Iran, Kashmar & Moshtaghi et al. (2016) \\
\hline ZMFUM2749 & KX219811 & Scarturus elater 1 & Iran, Golestan & Moshtaghi et al. (2016) \\
\hline ZMFUM2842 & KX219812 & Scarturus elater 1 & Iran, Bojnord & Moshtaghi et al. (2016) \\
\hline ZMFUM2862 & KX219813 & Scarturus elater 1 & Iran, Bojnord & Moshtaghi et al. (2016) \\
\hline ZMFUM1374 & KX219814 & Scarturus elater 1 & Iran, Kashmar & Moshtaghi et al. (2016) \\
\hline ZMFUM2897 & KX219815 & Scarturus elater 1 & Iran, Bojnord & Moshtaghi et al. (2016) \\
\hline ZMFUM2675 & JQ954927 & Scarturus elater 2 & Iran, Golestan & Dianat et al. (2013) \\
\hline ZMFUM2676 & JQ954929 & Scarturus elater 2 & Iran, Golestan & Dianat et al. (2013) \\
\hline ZMFUM2677 & JQ954930 & Scarturus elater 2 & Iran, Golestan & Dianat et al. (2013) \\
\hline ZMFUM2732 & KX219816 & Scarturus elater 2 & Iran, Golestan & Moshtaghi et al. (2016) \\
\hline ZMFUM2738 & KX219817 & Scarturus elater 2 & Iran, Golestan & Moshtaghi et al. (2016) \\
\hline ZMFUM2751 & KX219818 & Scarturus elater 2 & Iran, Golestan & Moshtaghi et al. (2016) \\
\hline ZMFUM3547 & KX219819 & Scarturus elater 2 & Iran, Sarakhs & Moshtaghi et al. (2016) \\
\hline ZMFUM3548 & KX219820 & Scarturus elater 2 & Iran, Sarakhs & Moshtaghi et al. (2016) \\
\hline ZMFUM3554 & KX219821 & Scarturus elater 2 & Iran, Sarakhs & Moshtaghi et al. (2016) \\
\hline ZMFUM3601 & KX219822 & Scarturus elater 2 & Iran, Torbate Jam & Moshtaghi et al. (2016) \\
\hline ZMFUM3602 & KX219823 & Scarturus elater 2 & Iran, Torbate Jam & Moshtaghi et al. (2016) \\
\hline ZMFUM3603 & KX219824 & Scarturus elater 2 & Iran, Torbate Jam & Moshtaghi et al. (2016) \\
\hline ZMFUM3604 & KX219825 & Scarturus elater 2 & Iran, Torbate Jam & Moshtaghi et al. (2016) \\
\hline ZMFUM3609 & KX219826 & Scarturus elater 2 & Iran, Torbate Jam & Moshtaghi et al. (2016) \\
\hline ZMFUM3613 & KX219827 & Scarturus elater 2 & Iran, Torbate Jam & Moshtaghi et al. (2016) \\
\hline Z & 3 & $\begin{array}{l}\text { Scarturus } \\
\text { euphratica }\end{array}$ & $\mathrm{n}$ & 013) \\
\hline
\end{tabular}




\section{Appendix. (Continued)}

\begin{tabular}{|c|c|c|c|c|}
\hline $\begin{array}{l}\text { Sample ID/ } \\
\text { Voucher } \\
\text { number }\end{array}$ & $\begin{array}{l}\text { Accession } \\
\text { Numbers }\end{array}$ & $\begin{array}{l}\text { Species/clades } \\
\text { Moshtaghi et al. } \\
\text { (2016) }\end{array}$ & Locality & Reference \\
\hline PMS18492 & KC465451 & S. williamsi & Lebanon & Kryštufek et al. (2013) \\
\hline- & KC465452 & S. williamsi & Lebanon & Kryštufek et al. (2013) \\
\hline DBSU10-245 & KC465442 & Scarturus euphratica & Turkey & Kryštufek et al. (2013) \\
\hline DBSU10-246 & KC465443 & Scarturus euphratica & Turkey & Kryštufek et al. (2013) \\
\hline DBSU10-247 & KC465444 & Scarturus euphratica & Turkey & Kryštufek et al. (2013) \\
\hline DBSU10-248 & KC465445 & Scarturus euphratica & Turkey & Kryštufek et al. (2013) \\
\hline GCSAR1936 & KC465446 & Scarturus euphratica & Syria & Kryštufek et al. (2013) \\
\hline GCSAR1948 & KC465447 & Scarturus euphratica & Syria & Kryštufek et al. (2013) \\
\hline GCSAR1949 & KC465448 & Scarturus euphratica & Syria & Kryštufek et al. (2013) \\
\hline GCSAR1954 & KC465449 & Scarturus euphratica & Syria & Kryštufek et al. (2013) \\
\hline GCSAR1962 & KC465450 & Scarturus euphratica & Syria & Kryštufek et al. (2013) \\
\hline ZMFUM2682 & JQ954939 & Scarturus hotsoni & Iran, Esfahan, Pukand & Dianat et al. (2013) \\
\hline ZMFUM2684 & JQ954940 & Scarturus hotsoni & Iran, Esfahan, Peykan & Dianat et al. (2013) \\
\hline ZMFUM2686 & JQ954941 & Scarturus hotsoni & $\begin{array}{l}\text { Iran, Esfahan, } \\
\text { Aminabad }\end{array}$ & Dianat et al. (2013) \\
\hline ZMFUM2687 & JQ954942 & Scarturus hotsoni & $\begin{array}{l}\text { Iran, Esfahan, } \\
\text { Aminabad }\end{array}$ & Dianat et al. (2013) \\
\hline ZMFUM2685 & JQ954943 & Scarturus hotsoni & Iran, Esfahan, Peykan & Dianat et al. (2013) \\
\hline ZMFUM1277 & JQ954960 & Scarturus hotsoni & Iran, Yazd & Dianat et al. (2013) \\
\hline ZMFUM1521 & JQ954961 & Scarturus hotsoni & Iran, Yazd & Dianat et al. (2013) \\
\hline ZMFUM1533 & JQ954962 & Scarturus hotsoni & Iran, Yazd & Dianat et al. (2013) \\
\hline ZMFUMT1045 & AJ389534 & Scarturus toussi East & Iran & Dianat et al. (2013) \\
\hline ZMFUM1431 & JQ954933 & Scarturus toussi East & Iran, Sarakhs & Dianat et al. (2013) \\
\hline ZMFUM1416 & JQ954938 & Scarturus toussi East & Iran, Cheshme Gilas & Dianat et al. (2013) \\
\hline ZMFUM2694 & JQ954954 & Scarturus toussi East & Iran, Cheshme Gilas & Dianat et al. (2013) \\
\hline ZMFUM2695 & JQ954955 & Scarturus toussi East & Iran, Cheshme Gilas & Dianat et al. (2013) \\
\hline ZMFUM2696 & JQ954956 & Scarturus toussi East & Iran, Cheshme Gilas & Dianat et al. (2013) \\
\hline ZMFUM1415 & JQ954957 & Scarturus toussi East & Iran, Cheshme Gilas & Dianat et al. (2013) \\
\hline ZMFUM1418 & JQ954958 & Scarturus toussi East & Iran, Cheshme Gilas & Dianat et al. (2013) \\
\hline ZMFUM2130 & JQ954959 & Scarturus toussi East & Iran, Sarakhs & Dianat et al. (2013) \\
\hline- & KX018304 & Scarturus toussi East & $\begin{array}{l}\text { Iran, Damghan, } \\
\text { Qusheh }\end{array}$ & $\begin{array}{l}\text { Mohammadi et al. } \\
\text { (2016) }\end{array}$ \\
\hline- & KX018305 & Scarturus toussi East & $\begin{array}{l}\text { Iran, Damghan, } \\
\text { Qusheh }\end{array}$ & $\begin{array}{l}\text { Mohammadi et al. } \\
\text { (2016) }\end{array}$ \\
\hline- & KX018306 & Scarturus toussi East & $\begin{array}{l}\text { Iran, Damghan, } \\
\text { Qusheh }\end{array}$ & $\begin{array}{l}\text { Mohammadi et al. } \\
\text { (2016) }\end{array}$ \\
\hline- & KX018307 & Scarturus toussi East & $\begin{array}{c}\text { Iran, Damghan, } \\
\text { Qusheh }\end{array}$ & $\begin{array}{l}\text { Mohammadi et al. } \\
\text { (2016) }\end{array}$ \\
\hline
\end{tabular}




\section{Appendix. (Continued)}

\begin{tabular}{|c|c|c|c|c|}
\hline $\begin{array}{l}\text { Sample ID/ } \\
\text { Voucher } \\
\text { number }\end{array}$ & $\begin{array}{l}\text { Accession } \\
\text { Numbers }\end{array}$ & $\begin{array}{c}\text { Species/clades } \\
\text { Moshtaghi et al. (2016) }\end{array}$ & Locality & Reference \\
\hline ZMFUM2864 & KX219828 & Scarturus toussi East & Iran, Bojnord & Moshtaghi et al. (2016) \\
\hline ZMFUM3557 & KX219829 & Scarturus toussi East & Iran, Sarakhs & Moshtaghi et al. (2016) \\
\hline ZMFUM3558 & KX219830 & Scarturus toussi East & Iran, Sarakhs & Moshtaghi et al. (2016) \\
\hline ZMFUM3553 & KX219831 & Scarturus toussi East & Iran, Sarakhs & Moshtaghi et al. (2016) \\
\hline ZMFUM2875 & KX219832 & Scarturus toussi East & Iran, Bojnord & Moshtaghi et al. (2016) \\
\hline ZMFUM1434 & KX219833 & Scarturus toussi East & Iran, Tabas & Moshtaghi et al. (2016) \\
\hline ZMFUM1438 & KX219834 & Scarturus toussi East & Iran, Sabzevar & Moshtaghi et al. (2016) \\
\hline ZMFUM3614 & KX219835 & Scarturus toussi East & Iran, Torbate Jam & Moshtaghi et al. (2016) \\
\hline ZMFUM2678 & JQ954934 & Scarturus toussi West & $\begin{array}{l}\text { Iran, Esfahan, } \\
\text { Mirabad }\end{array}$ & Dianat et al. (2013) \\
\hline ZMFUM2679 & JQ954935 & Scarturus toussi West & Iran, Tehran & Dianat et al. (2013) \\
\hline ZMFUM2680 & JQ954936 & Scarturus toussi West & Iran, Tehran & Dianat et al. (2013) \\
\hline ZMFUM2681 & JQ954937 & Scarturus toussi West & Iran, Tehran & Dianat et al. (2013) \\
\hline ZMFUM2680 & KX219836 & Scarturus toussi West & Iran, Tehran & Moshtaghi et al. (2016) \\
\hline ZMFUM4503 & KX219837 & Scarturus toussi West & Iran, Hamedan & Moshtaghi et al. (2016) \\
\hline ZMFUM2691 & JQ954947 & Scarturus williamsi & Iran, Hamedan & Dianat et al. (2013) \\
\hline ZMFUM2693 & JQ954949 & Scarturus williamsi & Iran, Zanjan & Dianat et al. (2013) \\
\hline ZMFUM2138 & JQ954951 & Scarturus williamsi & Iran, Ardebil & Dianat et al. (2013) \\
\hline DBSU10-235 & KC465439 & Scarturus williamsi & Turkey & Kryštufek et al. (2013) \\
\hline DBSU11-298 & KC465440 & Scarturus williamsi & Turkey & Kryštufek et al. (2013) \\
\hline DBSU11-299 & KC465441 & Scarturus williamsi & Turkey & Kryštufek et al. (2013) \\
\hline ZMFUM2688 & JQ954944 & Scarturus williamsi & $\begin{array}{l}\text { Iran, Chaharmahal } \\
\text { and Bakhtiari }\end{array}$ & Dianat et al. (2013) \\
\hline ZMFUM 2689 & JQ954945 & Scarturus williamsi & $\begin{array}{l}\text { Iran, Chaharmahal } \\
\text { and Bakhtiari }\end{array}$ & Dianat et al. (2013) \\
\hline ZMFUM2690 & JQ954946 & Scarturus williamsi & Iran, Hamedan & Dianat et al. (2013) \\
\hline ZMFUM2692 & JQ954948 & Scarturus williamsi & Iran, Zanjan & Dianat et al. (2013) \\
\hline ZMFUM2125 & JQ954950 & Scarturus williamsi & Iran, Ardebil & Dianat et al. (2013) \\
\hline ZMFUM2139 & JQ954952 & Scarturus williamsi & Iran, Ardebil & Dianat et al. (2013) \\
\hline
\end{tabular}

